\title{
A morte transmidiática de Osama bin Laden: remediação em jornalismo na cibercultura ${ }^{\mathrm{i}}$
}

\author{
Alan Mangabeira Mascarenhas \\ Universidade Federal da Paraíba - alanmangabeira@gmail.com \\ Mestrando do Programa de Pós-Graduação em Comunicação e \\ Culturas Midiáticas e Pesquisador do Grupo de Pesquisa em Processos \\ e Linguagens Midiáticas - Gmid/UFPB. \\ ANdRea Ferreira de Andrade Poshar FranÇa \\ Universidade Federal da Paraíba - andrea.poshar@gmail.com \\ Mestrando do Programa de Pós-Graduação em Comunicação e Culturas \\ Midiáticas e Pesquisador do Grupo de Pesquisa em Processos e \\ Linguagens Midiáticas - Gmid/UFPB. \\ MARCoS ANTONio Nicolau \\ Universidade Federal da Paraíba - marcosnicolau.ufpb@gmail.com \\ É professor do Departamento de Mídias Digitais e Coordena o Programa \\ de Pós-Graduação em Comunicação. Ensina e pesquisa comunicação e semiótica, \\ linguagens de jornalismo, mídias digitais e cibercultura, linguagem das marcas \\ e criação publicitária, história em quadrinhos.
}

\section{Resumo}

O jornalismo, constituído que é por narrativas diárias, tem sido palco de significativas transformações no contexto da cibercultura, sendo também lugar de fenômenos comunicacionais como a remediação e a narrativa transmidiática. O evento que envolveu a morte do terrorista Osama bin Laden, divulgado por um paquistanês no Twitter, surge como exemplo para uma tentativa de compreensão desses fenômenos. É o que se propõe o presente trabalho, a partir das definições de tais processos e sua aplicabilidade aos desdobramentos midiáticos da morte do terrorista.

\section{Palavras-chave}

Narrativas transmidiáticas. Pós-massivo. Cibercultura.

\begin{abstract}
Journalism, which is constituted by daily narratives, has been the stage of significant changes in the context of cyberculture, by revealing some communication phenomena such as transmedia storytelling and remediation. The event which involved the death of Osama bin Laden, released by a Pakistani on the Twitter, seems to be an example of an attempt to understand these phenomena. This work aims at showing such phenomena from the definitions of such processes and their applicability to media unrolling of the terrorist's death.
\end{abstract}

\section{Keywords}

Transmedia storytelling. Post-massive. Cyberculture. 
Q uando o paquistanês Sohaib Athar postou no Twitter que estava vendo helicópteros sobrevoando as proximidades da sua residência, não imaginava que estivesse anunciando um dos eventos jornalísticos de maior repercussão internacional dos últimos tempos: a invasão do esconderijo de Osama bin Laden e a morte do terrorista por forças militares norte-americanas.

Os desdobramentos midiáticos do fato, por sua vez, demonstram alguns dos fenômenos comunicacionais da atualidade, como a remediação (remediation) e a narrativa transmidiática: ambos aplicados aqui às estratégias de práticas jornalísticas, que hoje permeiam a cibercultura.

Pela perspectiva da remediação, ora estamos diante de plataformas diversas na internet, passando de um ambiente para outro em busca do que nos interessa, ora mergulhamos em uma narrativa que nos absorve a atenção, e esquecemos que estamos diante de um computador. Entre sites diversos, blogs e redes sociais, transitamos por notícias, conversas e curiosidades, mas sempre que encontramos uma boa história, real ou fictícia, entramos nela para nos envolver emocionalmente com sua trama e seus personagens.

Tais lógicas são denominadas, segundo os autores dos estudos sobre remediation, Bolter e Grusin (2000), de hipermediação e imediação. Na hipermediação há uma lógica de enaltecimento da presença do meio perante o indivíduo; na imediação, ao contrário, o meio fica "transparente", ou seja, não é perceptível, dado o envolvimento do usuário com a narrativa que se desenrola diante dos olhos e ouvidos.

Esse processo de remediação está diretamente relacionado com a narrativa transmidiática, responsável pela transição das histórias de uma mídia para outra com desdobramentos e continuidades informacionais que, muitas vezes, circulam entre o real e o fictício.

Trata-se de um fenômeno que ocorre sob duas perspectivas: as narrativas transmidiáticas e as estratégias transmidiáticas. No primeiro caso, temos em destaque um conteúdo que privilegia a narrativa; no segundo vemos a ubiqüidade de informações adicionais, mas não tão ligada a uma seqüência narrativa. 
A narrativa transmidiática, de acordo com Henry Jenkins (2008), é a prática de se contar uma história de forma fragmentada através de plataformas diversas para narrar partes diferentes de uma obra que juntas se complementarão. Mas, para o conceito de estratégias transmidiáticas, recorremos ao pesquisador e consultor de projetos transmidiáticos Geoffrey Long (2009), para quem o termo "transmídia” quer dizer qualquer coisa que se move de uma mídia para outra, independente do termo "narrativa": o caráter ubíquo da informação permite que esta se distribua por diferentes mídias convergentes e em processo de remediação.

Desse modo, todas as narrações envolvendo a morte de Osama bin Laden constituíram-se em evidente exemplo desses fenômenos comunicacionais e que nos exigem a compreensão conceitual e epistemológica dos processos de remediação e narrativa transmidiática, como parte de um esforço para compreendermos as práticas jornalísticas no contexto da cibercultura.

\section{O processo de remediação}

Ao afirmar que "o conteúdo de uma mídia é sempre outra mídia”, McLuhan (1964, p. 23) estava se referindo não só à mudança estrutural do conteúdo, mas a um processo mais complexo no qual, de fato, uma mídia é representada e incorporada à outra. Estas inter- relações dos meios, de acordo com o autor, "constitui um momento de verdade e revelação, do qual nasce a nova forma" (McLUHAN, 1964, p. 75).

Este fenômeno foi identificado pelos autores Bolter e Grusin (2000), anos mais tarde, como o processo de remediação (remediation). Tida pelos autores como uma das principais características das novas mídias digitais, a remediação é definida como a lógica formal com que um meio é capaz de apropriar-se das propriedades e peculiaridades de outro meio sendo este, seu antecessor.

A palavra "remediação" deriva do latim "remederi" que significa "curar, restaurar a saúde" ii e que, segundo os autores, melhor representa a forma com que um meio é visto pela sociedade - como uma mídia que renova (refashion) e proporciona novos usos (repurpose) às "velhas mídias" (older medium): 
O surgimento de todo novo meio é justificado porque ele preenche a lacuna ou repara a falha do seu antecessor, pois ele cumpre a promessa quebrada de uma velha mídia. (Tipicamente, é claro, os usuários não se dão conta de que a velha mídia falhou em sua promessa até o surgimento de um novo meio) ${ }^{\mathrm{iii}}$ (BOLTER; GRUSIN, 2000, p. 60)

Devemos aqui entender que, para os autores, "um meio é aquilo que remedia. Aquilo que se apropria das técnicas e significados sociais de outra mídia e cuja pretensão é dar-lhes vida ou repaginá-las em nome do real” iv (BOLTER; GRUSIN, 2000, p. 65). De fato, o desejo por se aproximar da realidade é uma das principais razões pelas quais os meios de comunicação alteram-se de forma tão espontânea e acelerada: quanto mais os modificarmos, maiores serão as probabilidades de atingirmos o real e, por conseguinte, o invisível.

"É como se cada tecnologia importante, antes de atingir níveis de saturação nas culturas, tenha tido de passar por dois estágios básicos: primeiro, estar em clara evidência; segundo, ser interiorizada até ao ponto de se tornar invisível" afirma Kerckhove (2009, p. 113). Para se tornar invisível e interiorizado, o meio precisa ser completamente "apagado" perante a presença do indivíduo. Tal fenômeno corresponderia ao que Bolter e Grusin (2000) identificaram como sendo a lógica da imediação (immediacy).

A transparência (transparency), como epistemologicamente definida pelos autores, retrata a ânsia que o usuário possui em "mergulhar" no conteúdo do meio, eliminando-o como intermediário deste contato, fazendo-o vivenciar o ambiente virtual como se, de fato, este fosse seu ambiente físico.

Umas das formas mais atuais que existem para atingir a imersão é, de acordo com Bolter (2006), a realidade mista. Esta não tem pretensão alguma de separar o que é virtual do real, mas sim em uni-los em prol da transparência - além do usuário "entrar" no mundo virtual, este também pode fazer o movimento inverso. Para o autor, a realidade mista é um híbrido entre o meio físico e virtual, cujo principal objetivo é proporcionar informações e experiências através de sons, imagens e textos digitalmente gerados para o mundo do usuário, reconfigurando a relação deste com seu entorno.

Neste contexto, podemos afirmar que entendemos a realidade a partir do conceito de Pereira (2008), que deve ser compreendida como um conjunto de padrões e 
sistemas estabelecidos que, juntos, organizam e orientam um determinado grupo em relação ao seu meio. É preciso ressaltar também que, em relação às realidades físicas ou não, o real não se opõe ao virtual.

Portanto, para gerar a transparência e imergir dentro de uma realidade que não a física, toda e qualquer tecnologia pode ser usada em seu favor. Para Bolter (2006, p. 110) “o objetivo da representação da transparência é o de eliminar as representações das tecnologias e suas técnicas e inserir o indivíduo em uma aparente relação não mediada com o objeto ou evento representado" v.

Porém, apesar da imediação buscar saciar o desejo do usuário pela experiência de vivenciar a imersão e eliminar toda e qualquer interferência do meio sobre esta, de tal forma que possa unificar e homogeneizar o espaço, não se pode negar a existência do meio - sem o qual a transparência, de fato, não poderia acontecer.

Isto nos leva à lógica da hipermediação que, segundo Bolter e Grusin (2000), enaltece a presença do meio perante o indivíduo, contrariando todo o processo de imediação.

A opacidade, como também a definem, tem por finalidade multiplicar o espaço, torná-lo heterogêneo e "janelado" (windowed style). Neste espaço, o usuário "oscila entre manipular janelas e examinar seu conteúdo, assim como oscila entre observar um hipertexto como uma textura de links e observar através destes para as unidades textuais como linguagens"vi (BOLTER; GRUSIN, 2000, p. 33). Apesar do estilo fragmentado, o propósito da hipermediação também é o de atingir a transparência e tornar este espaço multiplicado o mais "real" possível:

\begin{abstract}
A hipermediação e os meios transparentes são manifestações opostas do mesmo desejo: o desejo de romper os limites da representação para alcançar o real [...] A hipermediação busca o real multiplicando a mediação com intuito de criar uma sensação de plenitude, a saciedade da experiência, os quais podem ser tomados por realidade (BOLTER; GRUSIN, 2000, p. 53) $)^{\text {vii }}$.
\end{abstract}

Apesar de terem surgido no final do século XX, estas duas lógicas contrárias, porém, coerentes, persistem até hoje. Atualmente, tanto a imediação quanto a hipermediação são utilizadas como ferramentas para estratégias comunicacionais que visam, de alguma forma, capturar a atenção do indivíduo e direcioná-lo para o caminho 
do meio remediado. É o que afirma Grusin (JENKINS, 2011) ao dizer que a narrativa transmidiática pode ser um dos mais atuais exemplos de remediação já que envolve uma relação entre, pelo menos, duas mídias e que todos estes meios, podem ser vistos a serviço das lógicas da transparência e opacidade.

\section{Introdução às transmidiações}

Da fala às suas mediações observamos histórias serem contadas de formas diversas, acompanhando gradualmente a evolução das ferramentas de comunicação utilizadas pelo homem. Diante de um ambiente onde a telemática enfoca o digital, narrativas reconfiguram-se em um fluxo fragmentado e multisequencial através de mídias massivas e pós-massivas convergidas, com conteúdos remediados, incitando o que chamamos de transmidiações.

Este fenômeno se dá com narrativas transmidiáticas ou estratégias transmidiáticas, como veremos, ambas regidas pelo prefixo "trans", que indica "através de" ou "além de". No tocante às mídias, é relacionado à disposição de conteúdo, como quando temos a transposição da água (conteúdo) de um rio, alterando seu curso e fluxo, mas mantendo a composição do todo. Nas transmidiações, esta composição final vale mais que suas partes.

Transmidiações imbricam mídias de funções massivas e de funções pós-massivas. Sendo as funções massivas características de meios clássicos de comunicação, tais como a televisão e o rádio, enquanto as pós-massivas se ligam características das mídias digitais pelo seu caráter de leitura e escrita de informação dentro do mesmo espaço, como explica André Lemos (2007).

Para o autor, mídias de funções massivas adotam uma relação vertical com seu público, no qual os produtores de conteúdo regram o fluxo de seus produtos, buscando o controle e o lucro. Mídias clássicas como televisão, impressos, etc., obedecem a tais funções, que são características de uma era que começa na Revolução Industrial e pressupõem uma rede telemática inexistente, visando à criação de hits: "As funções massivas são aquelas dirigidas para a massa, ou seja, para pessoas que não se conhecem, que não estão juntas espacialmente e que assim têm pouca possibilidade de interagir" (LEMOS, 2007, p.6). 
A partir do final do século XX e início do XXI, podemos observar uma era pósindustrial e a necessidade de superação de tais modelos, guiados por transformações sócio-culturais que se tornam latentes com a disseminação de uma cultura eletrônica. Observamos então funções pós-massivas, as quais têm a rede telemática como potencializadora de suas ações, já que são baseadas em uma comunicação bidirecional. Para Lemos (2007, p.5), “as mídias de função pós-massiva, por sua vez, funcionam a partir de redes telemáticas em que qualquer um pode produzir informação, «liberando» o pólo da emissão, sem necessariamente haver empresas e conglomerados econômicos por trás".

As ferramentas interativas do ciberespaço atuam então como potencializadoras das ações do público diante do produto midiático e tais possibilidades se dão por três premissas dessa nova cultura: "A liberação da emissão, a conexão generalizada e a reconfiguração das instituições e da indústria cultural de massa" (LEMOS, 2007, p.6).

A partir destas premissas, tão distintas do que se vê em características de mídias clássicas, é que observamos uma reconfiguração das mídias e de seus conteúdos.

Por reconfiguração entendemos formas específicas pelas quais as mídias clássicas e seus produtos refazem-se para responder aos desafios propostos pelas características de uma cultura composta por mídias digitais.

Desta forma, as transmidiações são uma das consequências da reconfiguração das mídias diante da cultura do digital, já que denotam de uma rede telemática com ambiência de leitura e escrita que perpassam de produtos pós-massivos para produtos massivos.

\section{Estratégias e narrativas transmidiáticas}

O termo "transmidiação", que aqui destacamos, é fruto do conceito de narrativas transmidiáticas proposto por Jenkins (2008), que se desenvolve de forma bifurcada diante das práticas e usos observados nas experiências que envolvem tal fenômeno.

Para o autor, "uma história transmidiática se desenrola através de múltiplos suportes midiáticos, com cada novo texto contribuindo de maneira distinta e valiosa 
para o todo" (JENKINS, 2008, p. 135). Com plataformas nos oferecendo o melhor de si, é, então, criado um universo ficcional e imersivo para os fãs habitarem e interagirem.

Internacionalmente o modelo foi experimentado em séries como Lost, Kirill e Heroes, e nacionalmente na série Alice, que trazem, em maior ou menor nível, o público ao cerne da narrativa promovendo funções pós-massivas em produtos típicos da TV, uma mídia classicamente massiva. Jenkins destaca então a necessidade da inteligência coletiva, da rede telemática e da alta densidade do conteúdo para uma composição transmidiática:

Refere-se a uma nova estética que surgiu em resposta à convergência das mídias - uma estética que faz novas exigências dos consumidores e depende da participação ativa de comunidades de conhecimento. A narrativa transmidiática é a arte da criação de um universo. (JENKINS, 2008, p. 47).

É notória a vertente econômica por trás de qualquer ação transmidiática, principalmente quando uma das consequências desta é expandir franquias, tornando um produto ubíquo. Esta vertente e a popularização do gênero transmidiático nas narrativas geraram um uso indiscriminado do termo "transmídia" pelo mercado, tal como observamos acontecer com a interatividade, que hoje é apontada em qualquer situação onde o espectador tem mais de uma opção de escolha, mesmo que esta seja completamente controlada pelo produtor cultural.

Do mesmo modo, a vertente que aparece como consequência da prática e da apropriação do conceito proposto por Henry Jenkins se dá quando não nos referimos à narração transmidiática, mas a estratégias transmidiáticas. Para Geoffrey Long, em entrevista a Revista Pontocom (2009), "transmídia significa qualquer coisa que se move de uma mídia para outra". Tal colocação, diante dos fatos, nos leva a refletir transmidiações sem necessariamente estas serem extensões narrativas. $\mathrm{O}$ "transmidiático" aqui perde sua vertente de adjetivação, aparecendo apenas como "transmídia". Entende-se que toda narrativa transmidiática possui estas estratégias, mas que o contrário pode não acontecer.

Tais estratégias caracterizam-se pelo caráter ubíquo de uma informação, a exemplo, que se distribui por partes em diversas mídias convergentes que, por conseguinte, estão em processo de reconfiguração e remediação. É desta forma que 
podemos observar situações transmidiáticas quando saímos da ficção para fatos no jornalismo usualmente.

Ao ser questionado sobre as possibilidades transmídia no jornalismo, Jeff Gomez, responsável pelo filme Avatar e suas transmidiações, comentou viii que: "A essência é a habilidade de converter histórias, de um jeito que fará você esperar o comercial para ver o que acontece e até tomar ações", ao passo que ressalta a importância de um canal que estreite as relações entre o espectador e o jornalista.

Partindo do ponto de que este canal apenas é possível diante das funções pósmassivas, tentamos refletir a roteirização da notícia, mas principalmente como se dá o canal de ligação entre público e produtor quando o produto em questão é um fato e não uma realidade ficcional a ser construída e se estratégias e narrativas transmidiáticas são cabíveis neste espaço. Cabe ressaltar que adaptações da mesma informação não são consideradas como uma transmidiação da mesma.

\section{Um exemplo de jornalismo transmidiático}

Na madrugada do dia 2 de maio de 2011, o paquistanês Sohaib Athar presenciou um movimento estranho aos redores de sua casa e o publicou em seu Twitter, @ ReallyVirtual: "Helicóptero sobrevoando Abbottabad às 1AM (é um acontecimento raro)" "ix . Minutos depois, Athar volta a publicar: "Uma tremenda explosão sacudindo a janela. Espero que não seja o começo de algo desagradável :-S "x.

Descrevendo uma sucessão de acontecimentos que fugiam da normalidade de seu cotidiano, mas que até então não denunciavam nada grave, involuntariamente, o paquistanês narrava uma operação militar sigilosa dos Estados Unidos contra o líder da maior célula terrorista do mundo e responsável pelos ataques às Torres Gêmeas em 2001: Osama bin Laden.

Athar só veio ter conhecimento da relevância e magnitude de suas publicações horas após o ocorrido com uma nova publicação vinda diretamente do chefe de gabinete do ex Secretário de Defesa dos EUA, Keith Urban: "Fui informado por uma pessoa de confiança que mataram Osama bin Laden"’xi. 
Na tentativa de amenizar o estrondo causado com sua publicação, Urban volta a publicar vários outros tweets, nos quais ele confessa não saber se, até então, a informação era de fato oficial e que só o presidente dos EUA poderia se pronunciar em relação.

Porém, o movimento dentro das redes sociais aumentou com as publicações agora oferecidas tanto por informantes anônimos quanto do próprio governo americano, assim como também, de outros paquistaneses. Como um exemplo disso, temos o próprio Twitter do Sohaib Athar que, horas após seu relato, recebeu mais de 29.000 seguidores - e que aumentam até hoje.

O usuário paquistanês, que mantém desde 2007 um blog intitulado "Life in Pakistan" ("Vida no Paquistão", em tradução literal para o português), escreveu 23 mensagens para descrever os acontecimentos antes de descobrir do que se tratava de fato, ao passo que dialogava com outros na rede dando mais detalhes sobre explosões, helicópteros e pistas em geral sobre o acontecimento. Seus tweets eram então espalhados por outros usuários que tentavam também esclarecer as informações sem ligação direta com Osama bin Laden. Horas após o acontecimento, com mais informações divulgadas na mídia e o pronunciamento oficial do Presidente dos Estados Unidos, Athar comenta em sua página no Twitter: "Uh oh, agora eu sou o cara que contou em tempo real o ataque surpresa a Osama sem saber disso"xii.

O percurso das informações sobre a morte de Osama saiu do Twitter de forma desordenada para se organizar em sites de notícias e programas jornalísticos até o pronunciamento do presidente, onde o quebra-cabeça informacional ganha sua feição completa. Ou seja, onde o "todo" transmidiático se forma a partir das partes.

As mensagens de Athar, assim como de outros vizinhos do esconderijo de Osama, estavam disponíveis na rede para qualquer usuário que estivesse interessado em assuntos ligados ao teor internacional apresentado nas descrições. Era um espaço aberto à leitura e escrita, pós-massivo, onde se tinha a oportunidade de fazer uma "caça" a informações sobre o assunto e buscar mais detalhes.

Ainda após a conclusão dos fatos, o percurso transmidiático, fruto da convergência, que a informação percorreu estava devidamente arquivado na rede. Desta forma, mesmo os espectadores que tiveram acesso a informações mais completas 
através da televisão, a exemplo, poderiam retroceder, entrando por outras janelas deste universo transmidiático de informações e acompanhar os relatos de cidadãos comuns locais sem qualquer filtro por parte da imprensa em suas páginas no Twitter. Era possível também que espectadores mais interessados dialogassem em tempo real com os narradores dos fatos, contribuindo com informações mais detalhadas e direcionadas. Além de somar a estas informações, todo o material multimídia disponibilizado na web, como vídeos e fotos.

Passamos então a perceber características de uma ação transmidiática no jornalismo que se dá de forma involuntária. Ou seja, os dados se dispuseram de forma trasmidiática por natureza, transbordando de uma plataforma para outra, ao passo que portais de notícias como o The New York Times fizeram uso, citando a fonte, das informações publicadas por Athar no Twitter, assim como programas de televisão também o fizeram. Vemos uma narração transmidiática no fazer da notícia, algo outrora tão privado, mas que este se torna público ao leitor perante o hipertexto criado com as fontes que vieram do Twitter.

A narração dos fatos tem seu papel claro nas redes sociais, que incitam os usuários a narrarem partes do seu cotidiano com teor informacional relevante ou não. A ação da narrativa transmidiática aqui é involuntária por se tratar de um ato não planejado para a divulgação da notícia visando fins lucrativos em sua forma transmidiática. No mercado do entretenimento, a exemplo, produções de fãs referentes a um produto procedimental, como um filme, podem ativar este potencial.

Ainda assim, há uma das características principais na constituição de uma narrativa transmidiática que devem ser vistas aqui: a criação de um universo com janelas de entrada e saída para os diversos desdobramentos, ou apêndices, da história contada. Neste espaço, geralmente fictício, quando se trata de entretenimento, tende a possuir uma ficção coerente e imersiva, que simule de fato uma ambiência de realidade e propague a transparência do meio a fim de uma experiência realística.

A trajetória das informações sobre a morte de Osama bin Laden nos faz retomar o termo "detetives coletivos", usado em jogos ARG (Alternate Reality Games) e trazido por Henry Jenkins (2011), para ressaltar as interações sociais com outros leitores que se dão durante um evento transmidiático, proporcionado a "caça e vigia". Para Jenkins 
(2011), em publicação em seu blog ${ }^{\text {xiii }}$, "parte do prazer [da transmídia] é o que eu chamaria de "caça e vigia".

Esta situação de "caça" por parte do espectador no jornalismo, em que o usuário se torna seu próprio gatekeeper, é um dos principais nervos de uma rede transmidiática que narra um fato. Afinal, não é possível para o grande público dialogar com um repórter de uma grande emissora em tempo que a situação acontece, não proporcionando a imersão transparente necessária para a vivência de um universo do qual o usuário não pertence originalmente.

Nas descrições detalhadas de Athar e através do seu blog, ambos escritos horizontalmente com relação ao seu público, há um teor de imersão e diálogo necessário para a recriação deste universo que mantém a narrativa transmidiática coerente.

Nos estudos iniciais de Jenkins (2008), este universo era destacado como fictício, pois se tratava de narrativas com caráter de entretenimento, mas em casos jornalísticos destacamos a sua composição por fatos reais, enquanto a narração se dá através de diversas mídias. Tais mídias obedecendo a funções massivas e pós-massivas possibilitam ao público ligar-se em uma ação coletiva de caça e diálogo, não apenas para estruturar a notícia, mas principalmente na forma de consumo.

É aqui então que o leitor tem a possibilidade de ativar todo o potencial transmidiático contido numa narração, como a da morte de Osama bin Laden, e transformar o que poderia ser uma reportagem jornalística linear, em um evento transmidiático narrativo involuntário e ativo. Ao encontrar na reportagem jornalística, o endereço do perfil de Athar no Twitter, o leitor pode buscar o arquivo de informações adicionais ou conversar com a fonte, caso não tenha acompanhado em tempo real, fazendo esta ativação do potencial transmidiático.

Esta ação é composta por um sistema de imediação e hipermediação. O primeiro liga-se ao universo ficcional, ou real, que tende à imersão e, consequentemente, à transparência. O nível de detalhes e o tipo de narração da história, de forma igualitária com relação aos outros usuários da rede, contribuem nesse aspecto. Estar no mesmo patamar midiático que os indivíduos/leitores tende a tornar o envolvimento mais imersivo no universo do fato narrado. 
A hipermediação, por sua vez, caracteriza-se pela multiplicação de janelas para a composição de um universo de fatos, que aqui seriam fatos jornalísticos reais, os quais possibilitam ao indivíduo "caçá-las" ou, até mesmo, criar novas para poder, de alguma forma, atingir a imersão dentro da narrativa. Ou seja, por mais que o meio se faça presente perante o usuário, este buscará, dentre todas as janelas, obter um momento de transparência do conteúdo. Tal busca gera também situações de opacidade no caminho, nas quais o usuário sai de uma janela, mesmo que parcialmente, em busca de outra, e assim sucessivamente, em um movimento sem fim.

Esses momentos opacos podem ser "solucionados" assim que o usuário se deixa imergir em uma, entre tantas, janelas do universo transmidiático. Para Jenkins (2011) isto tem a ver com o nosso cotidiano, já que "como nossa realidade do dia após dia é formada pelas interações geradas através das mídias, as linhas entre a imediação e hipermediação ficam embasadas" xiv .

\section{Considerações finais}

Ao tratarmos da transmidiação de um fato concreto, ou seja, de uma notícia, dentro do jornalismo, o universo criado tende a ser composto por uma recriação do ambiente no qual se deu o fato. Temos então uma ambiência mediada propícia à imersão e imediação composta por funções massivas e pós-massivas. Através desta recriação, em que contamos com um jornalismo cidadão e colaborativo, é possível interação com as fontes da própria notícia.

O que Geoffrey Long (2009) trata por transmídia é demasiado elástico e pode ser naturalmente confundido com diversos conceitos. Apesar de representar várias ações do mercado sobre transmídia, acreditamos que mesmo diante de estratégias transmidiáticas, devemos ter resquícios de uma arquitetura narrativa em que cada novo desdobramento possa enriquecer o todo de forma notória, com informações adicionais importantes ao todo.

Se a transparência se dá através da "eliminação" do meio, o que devem ser revistos são o repórter clássico unilateral e plataformas exclusivamente massivas, permitindo que o indivíduo estabeleça um relacionamento direto com a história, 
sentido-a como se, de fato, estivesse participando ao vivo desta. Este relacionamento direto não exclui o papel do jornalista, apenas detona novas ferramentas jornalísticas no ato de narrar. Afinal, quanto maior forem os detalhes das informações obtidas e menor a presença do meio em si, maior será a probabilidade do indivíduo de "mergulhar" na história, transformando uma "mera" notícia pública em uma experiência individual e simulada.

De acordo com Bolter e Grusin (2000), o movimento destas lógicas da remediação não tem previsão de terem um fim. Muito pelo contrário, estarão sempre a serviço do indivíduo que procura, em uma missão também infindável, atingir a todo e qualquer custo, o mundo virtual dentro do seu mundo físico e vice-versa.

Nesse caso particular, envolvendo a operação que resultou na morte de Osama bin Laden pudemos verificar, por fim, não apenas como se mantém presentificada a lógica da remediação, mas também o processo que envolve as narrativas transmidiáticas nas mídias da atualidade. O elemento que permeia historicamente esse processo é a narrativa que, época após época, vem sendo moldada conforme as mudanças nos mecanismos de contar e recontar as histórias, lastreados agora pelos métodos não lineares engendrados pela Cibercultura.

\section{Referências}

BOLTER, Jay David; GRUSIN, Richard. Remediation: understanding new media. Cambridge: MIT Press, 2000.

BOLTER, Jay David. The desire for a transparency in an era of hybridity. 2006. Disponível em: $<$ http://pzwart3.wdka.hro.nl/mediawiki/images/5/56/User_Darija_Medic_ebook_desire_for_transparency. pdf $>$. Acesso em: 22 jul. 2011.

JENKINS, Henry. A Remediated, Premediated, and Transmediated: conversation with Richard Grusin. 2011. Disponível em: $<$ http://henryjenkins.org/2011/03/a remediated premediated and t.html $>$. Acesso em: 22 jul. 2011

. Cultura da Convergência. São Paulo: Aleph, 2008

KERCKHOVE, Derrik de. A pele da cultura: investigando a nova realidade eletrônica. São Paulo: Annablume, 2009.

LEMOS, André. Cidade e Mobilidade. Telefones Celulares, funções pós-massivas e territórios informacionais. In: Matrizes, Revista do Programa de Pós-Graduação em Ciências da Comunicação. USP, ano 1, n.1, São Paulo, 2007, pp.121-137. Disponível em

$<$ http://www.facom.ufba.br/ciberpesquisa/andrelemos/Media1AndreLemos.pdf $>$. Acesso em: 06 ago. de 2010 . 
Estudos em Jornalismo e Mídia - Vol. $9 \mathrm{~N}^{\circ} \mathrm{I}$ - Janeiro a Junho de 2012 ISSNe 1984-6924

LONG, Geoffrey. Entrevista à Revista Pontocom publicada online. 2009. Disponível em < http://www.revistapontocom.org.br/?p=1442>. Acesso em: 14 jun. 2011

McLUHAN, Marshall. Os meios de comunicação como extensões do homem. São Paulo: Cultrix, 1964.

PEREIRA, Vinicius Andrade. G.A.M.E.S.2.0: Gêneros e gramáticas de arranjos e ambientes midiáticos mediadores de experiências de entretenimento, sociabilidade e sensorialidades. Compós, 2008.

Disponível em: <http://www.compos.org.br/data/biblioteca_294.pdf>. Acesso em 30 jun. 2011. 


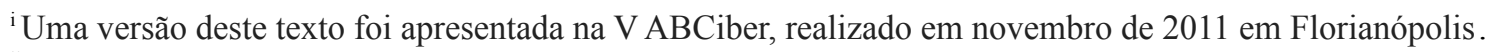

ii Tradução livre de "To heal, to restore the health" (BOLTER; GRUSIN, 2000, p. 59).

iii Tradução livre de "Each new medium is justified because it fills a lack or repairs a fault in its predecessor, because it fulfills the unkept promise of an older medium. (Typically, of course, users did not realize that the older medium had failed in its promise until the new one appeared)" (BOLTER; GRUSIN, 2000, p. 60).

iv Tradução livre de "A medium is that which remediates. Is is that which appropriates the techniques, forms and social significance of other media and attempts to rival or refashion them in the name of the real" (BOLTER; GRUSIN, 2000, p. 65).

v Tradução livre de "The goal of transparent representation is to efface the Technologies and techniques of representation and to place the viewer in an apparently un-mediated relationship to the objects or events represented" (BOLTER, 2006, p. 110).

vi Tradução livre de "Oscillates between manipulating the windows and examining their contents, just as she oscillates between looking at a hypertext as a texture of links and looking through the links to the textual units as languages" (BOLTER; GRUSIN, 2000, p. 33).

vii Tradução livre de "Hypermediacy and transparent media are opposite manifestations of the same desire: the desire to get part the limits of representation and to achieve the real [...] Transparent digital applications seek to get the real by bravely denying the fact of mediation, digital hypermediacy seek the real by multiplying mediation so as to create feeling of fullness, a satiety of experience, which can be taken by reality" (BOLTER; GRUSIN, 2000, p. 53).

viii Entrevista concedida em workshop na Rede Globo. Fonte:<http://www.tiagodoria.ig.com.br/2010/02/05/transmidiapode-ser-aplicada-ao-jornalismo/> Acesso em: 22 jul. 2011

ix Tradução livre de "Helicopter hovering above Abbottabad at $1 \mathrm{AM}$ (is a rare event)". Fonte:

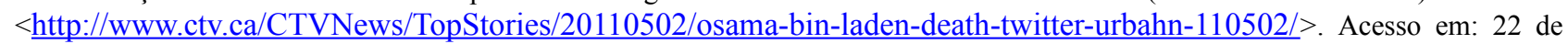
jul. 2011

x Tradução livre de "A huge window shaking bang here in Abbottabad Cantt. I hope it's not the start of something nasty :-S”. Fonte: idem. Acesso em: 22 jul. 2011

xi Tradução livre de "So I'm told by a reputable person they have killed Osama Bin Laden. Hot damn". Fonte:

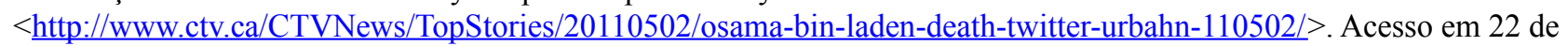
jul. 2011,

xii Tradução livre de: "Uh Oh, now I'm the guy who liveblogged the Osama raid without knowing it". Fonte: < http://www.digitaltrends.com/international/osama-bin-laden-death-raid-live-tweeted-by-accident/>. Acesso em $22 \mathrm{de}$ jul. 2011.

xiii Traduação livre de "Part of the pleasure is what I would call 'hunting and gathering"”. Fonte: $<$ http://henryjenkins.org/2011/03/a remediated premediated and t.html $>$. Acesso em: 22 jul. 2011

xiv Tradução livre de "as our everyday reality is shaped by our interactions through media, the lines between immediacy and hypermediacy blur”. Fonte: <http://henryjenkins.org/2011/03/a_remediated_premediated_and_t.html $>$ Acesso em: 22 jul. 2011 